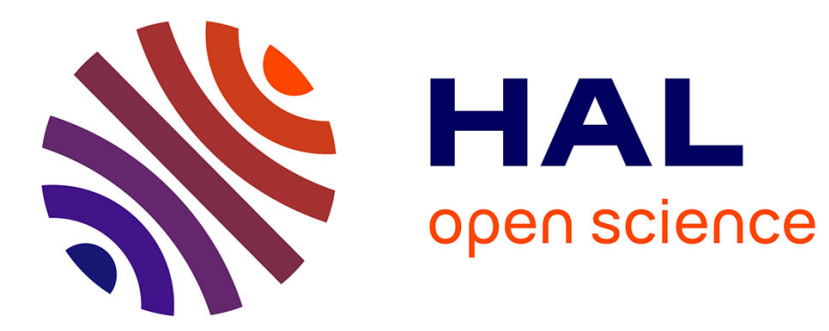

\title{
Broadband blazing with artificial dielectrics
}

\author{
Christophe Sauvan, Philippe Lalanne, Mane-Si Laure Lee
}

\section{To cite this version:}

Christophe Sauvan, Philippe Lalanne, Mane-Si Laure Lee. Broadband blazing with artificial dielectrics. Optics Letters, 2004, 29 (14), pp.1593-1595. hal-00858795

\section{HAL Id: hal-00858795 \\ https://hal-iogs.archives-ouvertes.fr/hal-00858795}

Submitted on 12 Sep 2013

HAL is a multi-disciplinary open access archive for the deposit and dissemination of scientific research documents, whether they are published or not. The documents may come from teaching and research institutions in France or abroad, or from public or private research centers.
L'archive ouverte pluridisciplinaire HAL, est destinée au dépôt et à la diffusion de documents scientifiques de niveau recherche, publiés ou non, émanant des établissements d'enseignement et de recherche français ou étrangers, des laboratoires publics ou privés. 


\title{
Broadband blazing with artificial dielectrics
}

\author{
Christophe Sauvan and Philippe Lalanne \\ Laboratoire Charles Fabry de l'Institut d'Optique, Centre National de la Recherche Scientifique, F-91403 Orsay Cedex, France
}

Mane-Si Laure Lee

Thales Research \& Technology, Domaine de Corbeville, 91404 Orsay Cedex, France

Received February 25, 2004

\begin{abstract}
The efficiency of conventional diffractive optical elements with échelette-type profiles drops rapidly as the illumination wavelength departs from the blaze wavelength. We use high dispersion of artificial materials to synthesize diffractive optical elements that are blazed over a broad spectral range $(\sim 1$ octave $)$ or for two different wavelengths. (c) 2004 Optical Society of America
\end{abstract}

OCIS codes: $050.1960,050.1950,050.1970$.

To maximize the efficiency with which a diffractive optical element (DOE) directs light into a chosen order of diffraction, it is necessary that it be blazed. ${ }^{1}$ The common way to achieve a blazing effect to first order is to manufacture continuous profiles such that a gradual phase variation of $2 \pi$ is introduced from one side of a Fresnel zone to the other. Échelette gratings or Fresnel lenses with triangular grooves are common examples that have been manufactured for the past 90 years. In the scalar approximation (valid for zone widths much larger than the wavelength), these DOEs provide a $100 \%$ diffraction efficiency at a particular wavelength $\lambda_{0}$ (the blaze wavelength). When these DOEs are illuminated at another wavelength, their efficiency drops, since the phase difference from one side of a zone to the other is no longer $2 \pi$. This drop in efficiency has a universal character, ${ }^{1}$ since it does not depend on the material used for the fabrication or on the phase function implemented, and results in the appearance of deterministic scattering into other diffraction orders, which represents a severe limitation for diffractive optical systems designed to operate in finite spectral bands. ${ }^{2}$ In this Letter we use the highly dispersive properties of artificial dielectrics, ${ }^{3,4}$ which are photonic crystal structures operating outside the bandgap, to achieve a blazing effect over a broad spectral range. The analysis is supported by rigorous electromagnetic computations and by an approximate model that provides evidence of the key role played by the high dispersion of artificial materials.

To illustrate our purpose, let us consider an artificial material composed of a square lattice of cylindrical air holes (diameter $d$ ) perforated in a nondispersive dielectric material with refractive index $n_{g}$. Provided that the periodicity constant $\Lambda_{s}$ is small enough compared with the wavelength of light, the subwavelength structures are not resolved by the light (in the sense of the far-field diffraction) and the heterogeneous structure behaves as a homogeneous material ${ }^{5}$ with an effective refractive index. In terms of Bloch waves, this means that all the Bloch modes supported by this periodic structure except the fundamental one are evanescent along the cylinder axis. ${ }^{6}$ The propagative nature of this mode is expressed as $\exp \left(j k_{0} n z\right)$, where $k_{0}$ is the wave-vector modulus in vacuum and $z$ is the propagation direction along the cylinder axis. The solid curve in Fig. 1 shows the effective index $n$ for $\Lambda_{s}=\lambda_{0} / n_{g}$ as a function of the ratio $\mathrm{d} / \Lambda_{s}$. More details concerning the calculation of the effective index can be found in Refs. 6 and 7. By controlling the local fraction of etched material, it is possible to implement a gradual variation of the effective index, and in turn to synthesize a gradual phase variation. DOEs relying on such artificial dielectrics that are graded from one side of a zone to the other were recently fabricated over small areas by use of electron-beam writing. Both pil$\operatorname{lar}^{6,7}$ and hole ${ }^{8}$ geometries have been reported. Not only have blazing effects been observed for both polarizations but also efficiencies superior to those of equivalent échelette-type DOEs have been obtained for small-period gratings ${ }^{6}$ and for diffractive lenses with a high numerical aperture. ${ }^{7,9}$

Generally, the synthesized effective indices range from some minimal value $n_{\min }\left(\lambda_{0}\right)$ to some maximal value $n_{\max }\left(\lambda_{0}\right)$, and first-order blazing at $\lambda=\lambda_{0}$

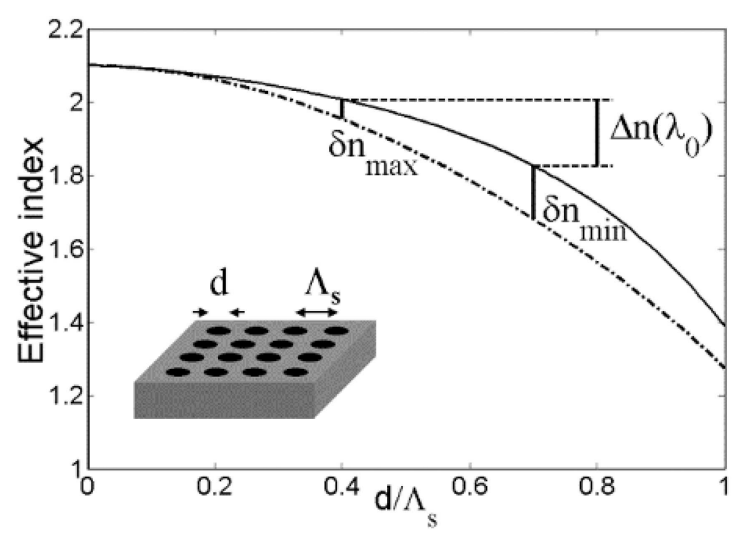

Fig. 1. Effective index of a square lattice of cylindrical air holes etched in a material with a refractive index $n_{g}=2.1$. Solid curve, $\lambda=\lambda_{0}=n_{g} \Lambda_{s}$; dashed-dotted curve, $\lambda \gg \Lambda_{s}$. The three quantities $\delta n_{\min }=n_{\min }\left(\lambda_{0}\right)-n_{\min }\left(\lambda_{\infty}\right), \delta n_{\max }=$ $n_{\max }\left(\lambda_{0}\right)-n_{\max }\left(\lambda_{\infty}\right)$, and $\Delta n\left(\lambda_{0}\right)$ involved in the important parameter $\alpha$ are shown.

(C) 2004 Optical Society of America 
is guaranteed, provided that the etched depth is $h=\lambda_{0} /\left[n_{\max }\left(\lambda_{0}\right)-n_{\min }\left(\lambda_{0}\right)\right]$. Phase transfer function $\phi\left(f, \lambda_{0}\right)$ of a DOE, where $f(x, y)$ is the local fraction of etched material $\left(f=\mathrm{d} / \Lambda_{s}\right.$ for hole geometries) at some $(x, y)$ point of the DOE surface is $\phi\left(f, \lambda_{0}\right)=k_{0}\left[n\left(f, \lambda_{0}\right)-n_{\min }\left(\lambda_{0}\right)\right] h$, where $n\left(f, \lambda_{0}\right)$ is the local effective index of the artificial material at $\lambda_{0}$. Illuminated at another wavelength $\lambda$, the artificial material disperses and a new range of effective indices is generated, as shown by the dashed-dotted curve in Fig. 1. The relationship between the phase transfer functions $\phi(f, \lambda)$ and $\phi\left(f, \lambda_{0}\right)$ is given by

$$
\phi(f, \lambda)=\frac{\lambda_{0}}{\lambda} \frac{n(f, \lambda)-n_{\min }(\lambda)}{n\left(f, \lambda_{0}\right)-n_{\min }\left(\lambda_{0}\right)} \phi\left(f, \lambda_{0}\right) .
$$

Without further assumptions, the calculation of the diffraction efficiency spectrum is a difficult task that can be attempted only numerically. However, under the assumption that the ratio $\left[n(f, \lambda)-n_{\min }(\lambda)\right] /\left[n\left(f, \lambda_{0}\right)-n_{\min }\left(\lambda_{0}\right)\right]$ depends only weakly on $f$ (this assumption will be validated below), an analytical treatment is made possible. When this ratio is identified to the value given for the local fraction of etched material corresponding to $n_{\max }\left(\lambda_{0}\right)$, the relationship between $\phi(f, \lambda)$ and $\phi\left(f, \lambda_{0}\right)$ becomes

$$
\phi(f, \lambda)=\frac{\lambda_{0}}{\lambda} \frac{\Delta n(\lambda)}{\Delta n\left(\lambda_{0}\right)} \phi\left(f, \lambda_{0}\right)
$$

where $\Delta n(\lambda)=n_{\max }(\lambda)-n_{\min }(\lambda)$. The linearity between $\phi(f, \lambda)$ and $\phi\left(f, \lambda_{0}\right)$ in Eq. (2) allows us to follow strictly the method developed in Ref. 1 for échelette-type DOEs with arbitrary phase functions. Following Ref. 1, we obtain for the first-order efficiency $\eta(\lambda)$

$$
\eta(\lambda)=\operatorname{sinc}^{2}\left[1-\frac{\lambda_{0}}{\lambda} \frac{\Delta n(\lambda)}{\Delta n\left(\lambda_{0}\right)}\right],
$$

where the function $\operatorname{sinc}(x)=\sin (\pi x) /(\pi x)$. Up to a third-order approximation in $\left(\Lambda_{s} / \lambda\right),{ }^{10}$ the dispersion relation of the artificial material can be written as

$$
n(\lambda)=n\left(\lambda_{\infty}\right)+n_{2}\left(\Lambda_{s} / \lambda\right)^{2}+\mathrm{O}\left(\Lambda_{s} / \lambda\right)^{4},
$$

where $n\left(\lambda_{\infty}\right)$ is the effective index of the artificial dielectric in the static limit $\lambda \rightarrow \infty$ and $n_{2}$ is a parameter depending on the local fraction $f$. By use of Eq. (4), $\Delta n(\lambda)$ can be written as

$$
\Delta n(\lambda)=\left[(1+\alpha)-\alpha\left(\lambda_{0} / \lambda\right)^{2}\right] \Delta n\left(\lambda_{0}\right),
$$

where $\alpha=\left\{\left[n_{\min }\left(\lambda_{0}\right)-n_{\min }\left(\lambda_{\infty}\right)\right]-\left[n_{\max }\left(\lambda_{0}\right)-\right.\right.$ $\left.\left.n_{\max }\left(\lambda_{\infty}\right)\right]\right\} / \Delta n\left(\lambda_{0}\right)$. Substituting $\Delta n(\lambda)$ from Eq. (5) into Eq. (3), one obtains, for the first-order diffraction efficiency at $\lambda$ of an artificial material DOE blazed at $\lambda_{0}$,

$$
\eta(\lambda)=\operatorname{sinc}^{2}\left[1-(1+\alpha)\left(\lambda_{0} / \lambda\right)+\alpha\left(\lambda_{0} / \lambda\right)^{3}\right] .
$$

Equation (6) shows that the spectral properties are completely determined by a single parameter $\alpha$ that depends on the artificial dielectric structure (geometry, lattice constant, index contrast, etc.) and on the important design parameters $n_{\min }\left(\lambda_{0}\right)$ and $n_{\max }\left(\lambda_{0}\right)$. The three physical quantities involved in $\alpha$ are shown in Fig. 1.

The important properties that are summarized in Fig. 2 are straightforwardly derived from Eq. (6). For any value of $\alpha, 1$ is a root of the third-order polynomial in $\lambda_{0} / \lambda$ in Eq. (6); i.e., the efficiency is $100 \%$ for the blaze wavelength $\lambda_{0}$. For $\alpha=0$, Eq. (6) becomes $\eta(\lambda)=\operatorname{sinc}^{2}\left[1-\left(\lambda_{0} / \lambda\right)\right]$ and corresponds to the classical formula for conventional échelette-type DOEs. ${ }^{1}$ For $\alpha \leq 0$ the polynomial has a single positive root $\lambda=$ $\lambda_{0}$ : there is a single wavelength $\lambda=\lambda_{0}$ for which artificial dielectric DOEs are blazed. More importantly, two different positive roots $\left(\lambda_{1}=\lambda_{0}\right.$ and $\left.\lambda_{2} \neq \lambda_{0}\right)$ exist for $\alpha>0$ : artificial dielectric DOEs are blazed for two different wavelengths. If these wavelengths are close to each other ( $\alpha$ is small), the component becomes blazed over a broad spectral range. Positive values of $\alpha$ are obtained for $n_{\min }\left(\lambda_{0}\right)-n_{\min }\left(\lambda_{\infty}\right)>n_{\max }\left(\lambda_{0}\right)-$ $n_{\max }\left(\lambda_{\infty}\right)$. This provides us with an important hint for designing broadband blazing effects: the artificial dielectric corresponding to $n_{\max }$ should be less dispersive than the one corresponding to $n_{\text {min }}$.

To test the validity of the approach presented above, we confronted the model predictions with rigorous electromagnetic computational results. For this purpose we designed an artificial dielectric grating composed of 35 subwavelength holes and 15 pillars patterned in a homogeneous material $\left(\mathrm{Si}_{3} \mathrm{~N}_{4}\right)$ with a refractive index $n_{g}=2.1$. The inset in Fig. 3 provides an illustration of the geometry along with the main grating parameters, $\Lambda_{s}=0.5 \lambda_{0}, \Lambda=25 \lambda_{0}$ and $h=\lambda_{0} / \Delta n\left(\lambda_{0}\right) \approx 1.8 \lambda_{0}$. The use of both pillar and hole geometries is motivated by the realization of relatively large $\alpha$ values (here $\alpha=0.39$ ) for a moderate aspect ratio (ratio of height $h$ to the smallest hole or pillar size), and the total number of features per period has been chosen so that the numerical computations present good convergences within the scalar approximation $(\Lambda \gg \lambda)$. Along the grating period, the local fraction of etched material is selected to generate a gradual effective-index variation from

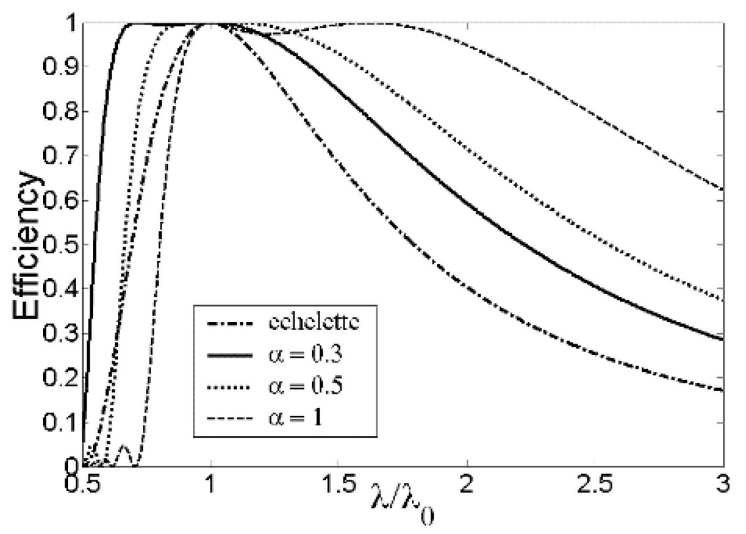

Fig. 2. Model predictions for the first-order diffraction efficiency of artifical dielectric DOEs for different values of $\alpha$. The dashed-dotted curve $(\alpha=0)$ corresponds to the efficiency of conventional échelette-type DOEs. 


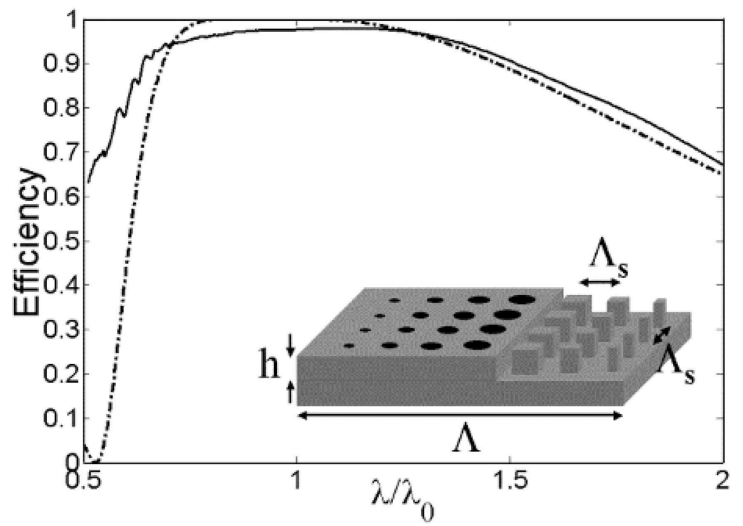

Fig. 3. Validation of the model: efficiency of a blazed binary grating for which $\alpha=0.39$ and comprising both cylindrical holes and square pillars. Solid curve, exact calculation; dashed-dotted curve, model prediction. Illustration of one grating period $\left(\Lambda=25 \lambda_{0}\right)$ composed of 35 holes and 15 pillars etched into a $\mathrm{Si}_{3} \mathrm{~N}_{4}$ film and illuminated from the $\mathrm{Si}_{3} \mathrm{~N}_{4}$ substrate at normal incidence. The sampling period is $\Lambda_{s}=0.5 \lambda_{0}$.

$n_{\min }\left(\lambda_{0}\right) \approx 1.57$ (pillar width of $\left.0.34 \lambda_{0}\right)$ to $n_{\max }\left(\lambda_{0}\right)=n$ (holes with null diameter). Once the grating has been designed, the grating efficiencies in all transmitted orders are calculated with the Fourier modal method. ${ }^{11}$ For the calculation, $111 \times 11$ Fourier harmonics are retained. We estimate that the absolute computational accuracy of all efficiencies is better than 0.01 . The grating is assumed to be illuminated under normal incidence from the substrate $\left(n_{s}=n_{g}=2.1\right)$ with unpolarized light. The first-order efficiency (normalized to the total energy diffracted in all transmitted orders) is shown in Fig. 3, together with the model predictions for $\alpha=0.39$. Good agreement over the full spectrum is obtained, showing a blazing effect $(\eta>95 \%)$ over more than 1 octave. However, there are also discrepancies. At blaze wavelength $\lambda_{0}$, the calculated efficiency does not reach unity. The $2.5 \%$ deviation results from a slight shadowing effect $^{9}(<1 \%)$ because of the finite grating period and from a high-order scattering effect $(\approx 1.5 \%)$ that is due to a loss of validity of the artificial dielectric approximation and geometry in a small zone at the boundary between holes and pillars. These effects are expected to vanish for larger periods $\Lambda$. For $\lambda<0.7 \lambda_{0}$, the model underestimates the efficiency and does not predict some small oscillations. For these small wavelengths, not only does the third-order approximation in Eq. (4) underestimate the high dispersion of artificial dielectrics but also the artificial dielectric model ceases to be valid, since the subwavelength structures support more than one propagative Bloch mode $^{6}$ for some $f$ values.

Until now the intrinsic dispersion of the base dielectric material has been neglected. For small values of $\lambda\left(\lambda<1.5 \lambda_{0}\right)$, the artificial material is highly dis- persive and the intrinsic dispersion can be neglected. For example, the relative refractive-index variation of $\mathrm{Si}_{3} \mathrm{~N}_{4}$ is only $1 \%$ over 1 octave in the visible region of the spectrum, whereas the relative effective-index variation of the artificial material used in Fig. 3 can be as high as $20 \%$. For large values of $\lambda$, the artificial material is weakly dispersive, see Eq. (4), and the effect of the intrinsic dispersion is likely to be nonnegligible. Since the refractive index generally decreases as the wavelength increases, we anticipate an improvement of the diffraction efficiency in comparison with our predictions.

An approximate model has been introduced to investigate the spectral behavior of DOEs composed of highly dispersive artificial materials implementing gradual effective-index variations from one side of a zone to the other. The model predicts unique spectral properties that significantly differ from those of conventional échelette-type DOEs, showing the possibility of designing DOEs with high efficiencies (>95\%) over nearly 1 octave. The predictions have been validated by rigorous electromagnetic computations. This finding may have important applications for diffractive optical systems designed to operate in finite spectral bands, especially in near-infrared bands II and III, for which standard fabrication processes allow the manufacture of deep etches in semiconductors with high refractive indices, i.e., artificial dielectrics with high dispersion.

C. Sauvan (christophe.sauvan@iota.u-psud.fr) is grateful to the Délégation Génération de l'Armement for his Ph.D. fellowship.

\section{References}

1. G. J. Swanson, "Binary optics technology: the theory and design of multilevel diffractive optical elements," MIT Tech. Rep. 854 (Massachusetts Institute of Technology, Cambridge, Mass., 1989).

2. D. A. Buralli and G. M. Morris, Appl. Opt. 31, 4389 (1992).

3. S. Y. Lin, V. M. Hietala, L. Wang, and E. D. Jones, Opt. Lett. 21, 1771 (1996).

4. M. Notomi, Phys. Rev. B 62, 10696 (2000).

5. A. A. Krokhin, P. Halevi, and J. Arriaga, Phys. Rev. B 65, 115208 (2002).

6. Ph. Lalanne, S. Astilean, P. Chavel, E. Cambril, and H. Launois, Opt. Lett. 23, 1081 (1998).

7. Ph. Lalanne, S. Astilean, P. Chavel, E. Cambril, and H. Launois, J. Opt. Soc. Am. A 16, 1143 (1999).

8. F. T. Chen and H. G. Craighhead, Opt. Lett. 20, 121 (1995).

9. L. Lee, Ph. Lalanne, J. C. Rodier, P. Chavel, E. Cambril, and Y. Chen, J. Opt. A Pure Appl. Opt. 4, S119 (2002).

10. Ph. Lalanne and D. Lalanne, J. Mod. Opt. 43, 2063 (1996).

11. L. Li, J. Opt. Soc. Am. A 14, 2758 (1997). 